\title{
Interactions between epidermal growth factor receptor tyrosine kinase inhibitors and proton-pump inhibitors/histamine type-2 receptor antagonists in non-small cell lung cancer: a systematic review and meta-analysis
}

\author{
Wilson Sim ${ }^{1}$, Sneha Rajiv Jain ${ }^{1}$, Wen Hui Lim ${ }^{1}$, Yip Han Chin ${ }^{1}$, Cheng Han Ng $^{1}$, Nicholas Syn ${ }^{1}$, \\ Kang Shiong Goh ${ }^{2}$, Ross Soo ${ }^{3}$, Lingzhi Wang ${ }^{1,4}$, Boon Cher Goh ${ }^{1,3,4}$ \\ ${ }^{1}$ Yong Loo Lin School of Medicine, National University of Singapore, Singapore, Singapore; ${ }^{2}$ Department of Internal Medicine, National University \\ Health System, Singapore, Singapore; ${ }^{3}$ Department of Haematology-Oncology, National University Cancer Institute, Singapore, Singapore; ${ }^{4}$ Cancer \\ Science Institute of Singapore, National University of Singapore, Singapore, Singapore \\ Contributions: (I) Conception and design: N Syn, KS Goh, R Soo, L Wang, BC Goh; (II) Administrative support: N Syn, KS Goh, R Soo, L Wang, \\ BC Goh; (III) Provision of study materials or patients: N Syn, L Wang, BC Goh; (IV) Collection and assembly of data: W Sim, SR Jain, WH Lim, \\ YH Chin, CH Ng, N Syn, KS Goh; (V) Data analysis and interpretation: W Sim, SR Jain, WH Lim, YH Chin, CH Ng, N Syn, KS Goh; (VI) \\ Manuscript writing: All authors; (VII) Final approval of manuscript: All authors. \\ Correspondence to: Nicholas Syn. Dean's Fellow, Yong Loo Lin School of Medicine, National University of Singapore, Singapore, 10 Medical Dr, \\ Singapore 117597. Email: nicholassyn@gmail.com; Boon Cher Goh. Senior Consultant, Department of Haematology-Oncology, National University \\ Cancer Institute, Singapore; Group Chief, Physician Leadership and Organisation Development Office, National University Health System (NUHS); \\ Deputy Director (Research), National University Cancer Institute, Singapore (NCIS); Director, Investigational Medicine Unit, NUHS; Deputy \\ Director, Cancer Science Institute of Singapore, NUS; Professor, Department of Pharmacology and Medicine, NUS; 5 Lower Kent Ridge Rd, \\ Singapore 119074. Email: phcgbc@nus.edu.sg; Ross Soo. Senior Consultant, Department of Haematology-Oncology, National University Cancer \\ Institute, Singapore; Adjunct Senior Research Fellow, Cancer Science Institute, National University of Singapore (NUS), 5 Lower Kent Ridge Rd, \\ Singapore 119074. Email: ross_soo@nuhs.edu.sg.
}

Background: Epidermal growth factor receptor (EGFR) tyrosine kinase inhibitors (TKIs) are increasingly used for advanced non-small cell lung cancer (NSCLC) as first-line therapy. The bioavailability and efficacy of oral EGFR-TKIs could be affected by acid suppression (AS) therapy such as PPIs and H2RAs which are reported to be over-prescribed. Hence, there is a need to investigate the effect of AS on the overall survival (OS), progression-free survival (PFS) and adverse effect profile in patients treated with EGFR TKIs.

Methods: An electronic database search of Medline and Embase was performed following PRISMA guidelines on 17 January 2021. Studies analyzing interactions between EGFR TKIs and PPIs/H2RAs in NSCLC patients were included. Abstracts, non-English or non-Japanese studies or studies using nonEGFR TKIs were excluded. Hazard ratios (HRs) were pooled using generic inverse variance random effects model. Effect sizes for dichotomous variables were pooled using Mantel-Haenszel random effects model. Significance was considered at $\mathrm{P} \leq 0.05$. Heterogeneity was assessed with Cochran Q-test and I2 test. Publication bias was assessed with funnel plots. The assessment of quality and risk of bias of randomized and non-randomized studies were undertaken with RoB 2 and the ROBINS-I tool respectively.

Results: Out of 1,173 potentially relevant articles, 14 articles were included in the final analysis. The pooled prevalence of AS in patients taking EGFR TKI was 30.71\% in 4,010 individuals. Patients who were treated only with EGFR TKI had significantly better OS (HR =1.46, 95\% CI: $1.27-1.72 ; \mathrm{P}<0.00001)$ and PFS (HR =1.63, 95\% CI: 1.35-1.98; P<0.00001). The OS for EGFR mutation positive patients only was as similarly significant as the OS in all patients taking EGFR TKI, while the PFS in mutation positive patients was significantly worsened with AS. PPIs resulted in a significantly worsened OS and PFS but H2RAs did not produce significantly different OS and PFS between AS and non-AS users. There were no significant differences in the incidence of rash $(\mathrm{OR}=0.81,95 \% \mathrm{CI}: 0.50-1.32 ; \mathrm{P}=0.40)$, diarrhoea 
$(\mathrm{OR}=1.03,95 \%$ CI: $0.63-1.67 ; \mathrm{P}=0.91)$ or other adverse effects.

Conclusions: Co-administration of AS medications with first-generation EGFR-TKIs in NSCLC worsens survival outcomes. Physicians should only prescribe AS medications when absolutely clinically indicated.

Keywords: Epidermal growth factor receptor (EGFR); tyrosine kinase inhibitors (TKI); acid suppression; drug interaction

Submitted May 07, 2021. Accepted for publication Jun 18, 2021.

doi: $10.21037 /$ tlcr-21-378

View this article at: https://dx.doi.org/10.21037/tlcr-21-378

\section{Introduction}

With lung cancer being the leading cause of cancer related deaths worldwide (1), the epidermal growth factor receptor (EGFR) has been found to play a significant role in the development of non-small cell lung cancer (NSCLC) (2). Recent guidelines have put forth systemic treatment regimens involving EGFR-tyrosine kinase inhibitors (TKIs), including erlotinib, gefitinib, afatinib, dacomitinib and osimertinib, as the first line therapy for advanced NSCLC harbouring sensitizing EGFR mutations (exon 19 deletions, exon 21p.L858R point mutation) (3). The use of EGFR TKIs has also been indicated as an adjuvant therapy for stage IIB, IIIA or high risk stage IB, IIA EGFR mutation positive NSCLC patients (3). The superiority of EGFR-TKIs over the conventional platinumbased doublet chemotherapy have been demonstrated by an improved response and progression-free survival (PFS) in large, randomized trials $(4,5)$.

The bioavailability of the most widely used firstgeneration EGFR TKIs (erlotinib and gefitinib) are dependent on the gastric acidity and absorption in the stomach, raising questions about the possible effect of co-administering medications that raise gastric $\mathrm{pH}$ $(6,7)$. Concurrent omeprazole administration has been shown to reduce erlotinib area under curve (AUC) and maximum concentration (Cmax) by about $46 \%$ and $61 \%$, respectively (8). Similarly, gefitinib has a $\mathrm{pH}$-dependent solubility and AUC and Cmax could decrease by up to $44 \%$ and $70 \%$ respectively (8). The pharmacokinetics of another widely used third-generation EGFR TKI osimertinib has not been shown to be affected by AS in an open-label study of healthy male volunteers (6).

Acid suppression (AS) medications are among the most common drug classes used in the world and are also available as over-the-counter medications (9). Among which, proton-pump inhibitors (PPIs) and histamine type-2 receptor antagonists (H2RAs) are the most commonly used in AS therapy which aims to maintain an intragastric $\mathrm{pH}$ above 4 (10-12). Consequently, the absorption, AUC and Cmax of erlotinib and gefitinib could be drastically reduced.

AS therapy is often prescribed as prophylaxis in patients with NSCLC treated with corticosteroids and has reported to be over-prescribed for therapeutic or prophylactic purposes (13), with a given prevalence of $33.2-46.3 \%$ of lung cancer patients concurrently using AS (14). Given the widespread AS use, a significant proportion of NSCLC patients may be receiving EGFR-TKI and AS therapy concurrently (14). Hence, there is a need to investigate the effect of AS on EGFR TKI efficacy, investigating its impact on overall survival (OS), PFS and its side effect profile.

We present the following article in accordance with the Preferred Reporting Items for Systematic Reviews and Meta-analysis (PRISMA) reporting checklist (available at https://dx.doi.org/10.21037/tlcr-21-378).

\section{Methods}

\section{Search strategy}

The Preferred Reporting Items for Systematic Reviews and Meta-analysis (PRISMA) guidelines was used in the synthesis of this review (15) with the PRISMA checklist completed. An electronic database search of Medline and Embase was performed on 17 January 2021 using keywords and terms synonymous with 'EGFR, 'TKI', 'Acid Suppression' and 'Drug Interaction'. An example of the search strategy can be found in the Table S1. The references of included articles were also assessed for suitability for inclusion. 


\section{Study selection and data extraction}

The inclusion criteria comprised comparative studies, that focused on the effects of EGFR TKIs (predominately firstgeneration erlotinib and gefitinib) in NSCLC patients on PPIs/H2RAs versus NSCLC patients not on AS therapy. A variety of quantitative methodology, such as randomized controlled trials (RCT) and cohort studies, was selected. Exclusion criteria included the use of nonEGFR TKIs, abstracts and studies that were non-English or non-Japanese. Article selection was performed by two authors, using the inclusion and exclusion criteria and any discrepancies were resolved based on the consensus with a third author. The blinded pair then extracted details such as the author, year of publication, title, country of origin, study design and duration, number and demographics of subjects, OS, PFS and adverse effects of the two drugs. When mean and standard deviation data were not reported, transformation of existing values was performed using existing methods $(16,17)$. If hazard ratios (HRs) were not provided, they were estimated from the log-rank p value, the median time-to-event, and time-point survival rates, using methods from Parmar et al. (18).

\section{Statistical analysis and quality assessment}

Analysis was done in Revman 5.4 and R studio (Version 1.3.1093). A single arm analysis was used to pool the proportion of NSCLC patients on PPI and H2RA using the generalized linear mixed model (GLMM) with ClopperPearson intervals to stabilize the variance $(19,20)$. For timeto-event variables, hazard ratios (HRs) were pooled using the generic inverse variance method with a random effects model for both OS and PFS. A sensitivity analysis based on the EGFR mutation status was performed to observe the effects of only mutant NSCLC. Next, a subgroup analysis based on the type of acid suppression (AS) used was performed to compare the effect size between PPI and H2RAs. Effect sizes for dichotomous variables were pooled using Mantel-Haenszel random effects model. Significance was considered at $\mathrm{P} \leq 0.05$. When there was insufficient data amount for meta-analysis, a descriptive approach was undertaken for the presentation of findings (this was performed for AEs). Heterogeneity was assessed with Cochran Q-test and $\mathrm{I}^{2}$ test, with a significance value of at $\mathrm{P} \leq 0.10$ or $\mathrm{I}^{2} \geq 40$ respectively $(21,22)$. Publication bias was assessed with funnel plots when sufficient studies were available $(\mathrm{n}>10)(23,24)$. Publication bias was assessed using visual inspection of funnel plots.

The assessment of quality and risk of bias of randomized and non-randomized studies were undertaken with Risk of Bias 2 (RoB 2) (25), and the ROBINS-I tool (26) respectively. RoB 2 assesses the risk of bias from the randomization process, deviations from intended interventions, missing outcome data, measurement of the outcome and in selection of the reported result. The ROBINS-I tool assesses bias due to confounding, selection, classification of interventions, deviations from intended interventions, missing data, measurement of outcomes and in reporting results.

\section{Results}

The search strategy yielded 1,173 potentially relevant articles. After titles and abstracts screened, 35 full texts were reviewed, of which 14 were included in the final analysis (Figure 1). Chu et al. (27) was excluded as the study evaluated patients with advanced gastroesophageal cancer and patients were under concurrent capecitabine and oxaliplatin (CapeOx) chemotherapy and capecitabine may increase plasma concentration of erlotinib (28), hence complicating the potential effects of acid suppression (AS) on EGFR TKIs. In total, 1,197 patients were treated with EGFR TKI together with AS and 3,298 patients were treated with EGFR TKI only. Of the included studies, 13 were retrospective cohort studies while one was a retrospective analysis of the BR.21 phase III clinical trial (29). Four studies analyzed patients treated with first-line therapy, 6 studies analyzed patients with at least 1 prior treatment, while the remaining 4 did not report the line of therapy. The characteristics of included articles including patient demographics, details of AS and EGFR TKI treatment and risk of bias assessment can be found in Table 1 .

\section{Proportion of patients with concurrent AS}

The overall pooled prevalence of AS in patients taking EGFR TKI was $30.71 \%$ (95\% CI: $23.28-39.31 \%$; Figure 2) in 4,010 individuals. A sensitivity analysis was conducted to observe the rate of PPI and H2RA in NSCLC. The prevalence of PPI in EGFR TKI patients was 19.33\% (95\% CI: 13.01$27.73 \%$ ), while the prevalence of H2RA was $25.13 \%$ (95\% CI: $14.76-39.42 \%)$.

\section{Survival outcomes}

Patients who were treated only with EGFR TKI had 


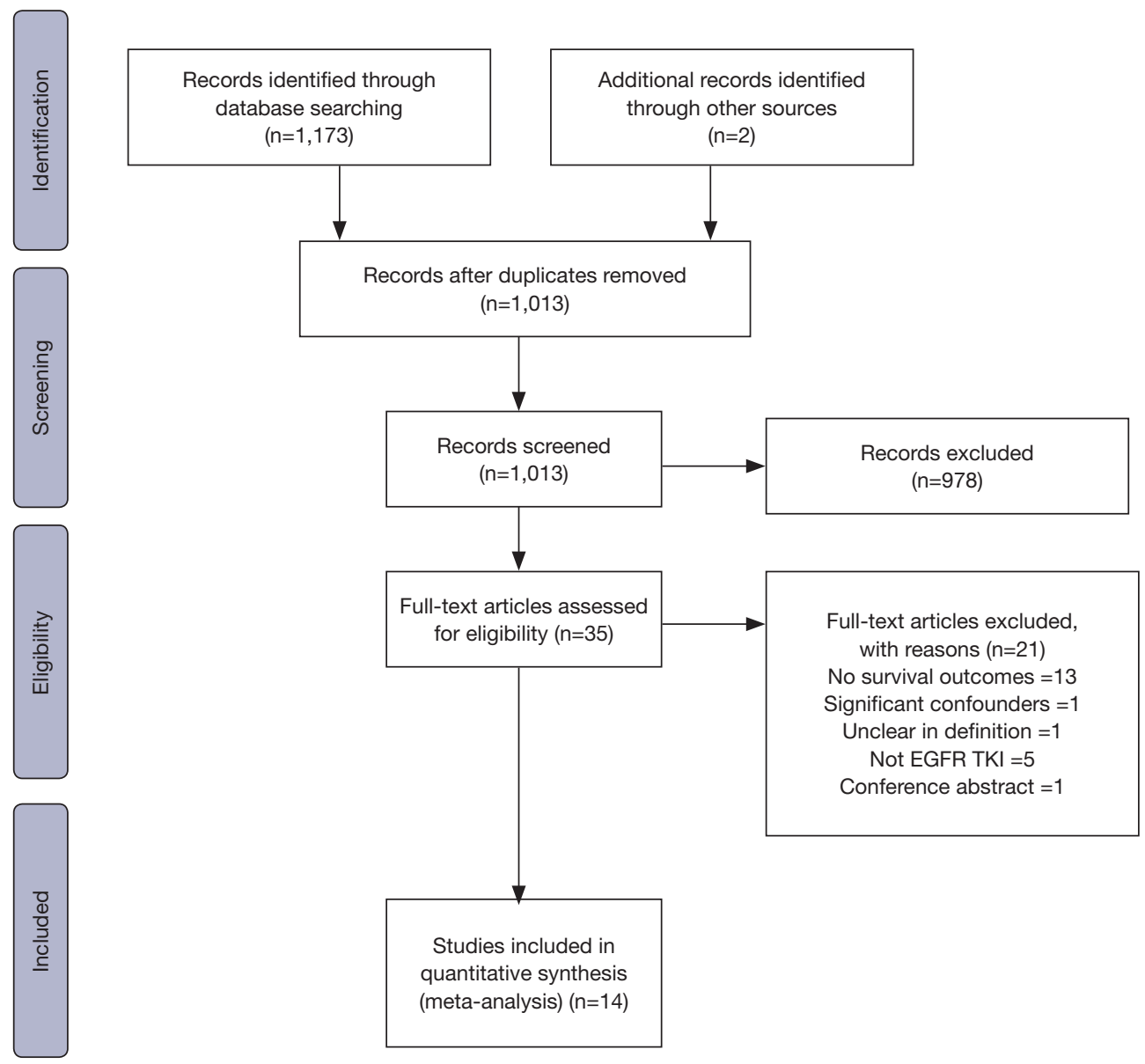

Figure 1 PRISMA flow diagram of included articles.

significantly better OS (HR $=1.46,95 \%$ CI: $1.27-1.72$; $\mathrm{P}<0.00001$; Figure 3) in 3,694 patients. The funnel plot for OS was symmetrical (Figure S1). Similarly, PFS favored patients who were treated with EGFR TKI only (HR $=1.63$, 95\% CI: $1.35-1.98 ; \mathrm{P}<0.00001 ;$ Figure 4$)$ in 2,433 patients. The funnel plot for PFS was symmetrical revealing no publication bias (Figure S1). A sensitivity analysis was conducted to include only EGFR mutation positive patients. The OS for EGFR mutation positive patients among 2,544 patients was as similarly significant as the OS for all patients taking EGFR TKI (HR $=1.50$, 95\% CI: 1.13-1.99, $\mathrm{P}=0.005$; Figure 5). However, AS significantly worsened the PFS among 350 EGFR mutation positive patients ( $\mathrm{HR}=2.19,95 \% \mathrm{CI}: 1.34-3.59$, $\mathrm{P}=0.002$; Figure 6).

A subgroup analysis was conducted to compare OS and PFS between PPI and H2RA groups. The OS HR for PPI was 1.98 (95\% CI: $1.33-2.94, \mathrm{P}=0.0007)$ in 1,114 patients and the OS HR for H2RA was 1.04 (95\% CI: 0.70-1.55, $\mathrm{P}=0.28)$ in 253 patients. There was significant difference between the two groups $(\mathrm{P}=0.03)$. Similarly, the PFS for PPI in 159 patients was significantly different $(\mathrm{HR}=3.39$, 95\% CI: 2.18-5.26, P<0.00001) The PFS for H2RA was however, not significantly different in 253 patients ( $\mathrm{HR}=1.48,95 \% \mathrm{CI}: 0.63-3.49, \mathrm{P}=0.37)$. The subgroup difference was not statistically significant $(\mathrm{P}=0.09)$.

\section{Adverse effects}

There were no significant differences in the incidence of rash (OR $=0.81,95 \%$ CI: $0.50-1.32 ; \mathrm{P}=0.40 ;$ Figure 5) and diarrhoea $(\mathrm{OR}=1.03,95 \% \mathrm{CI}: 0.63-1.67 ; \mathrm{P}=0.91$; Figure 7) between AS and non-AS users. Other adverse effects reported include vomiting (30), loss of appetite (30), oral ulcers (30), stomatitis (31), elevated aminotransferase (AST/ALT) (30,31), interstitial lung disease (30-32), were 


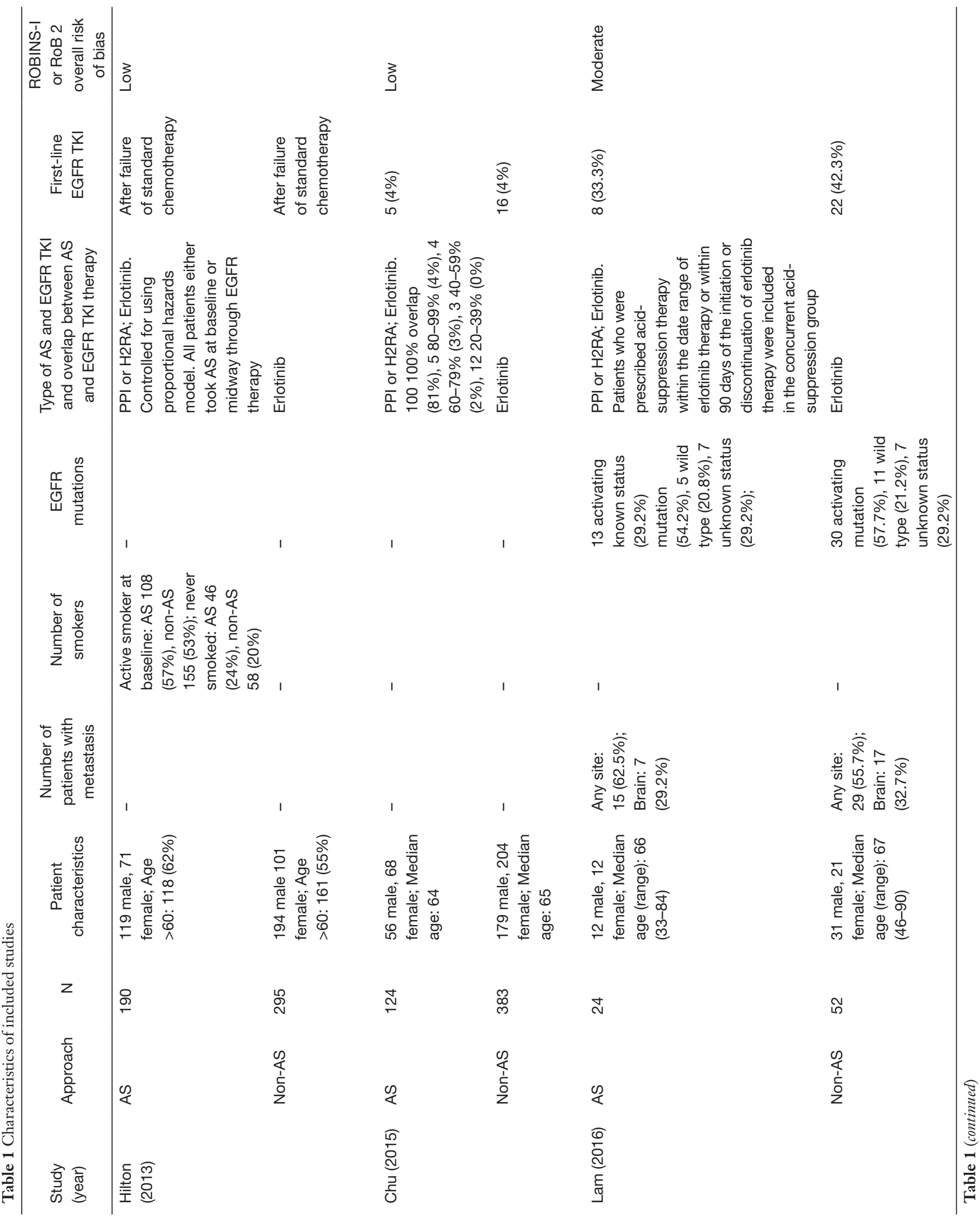




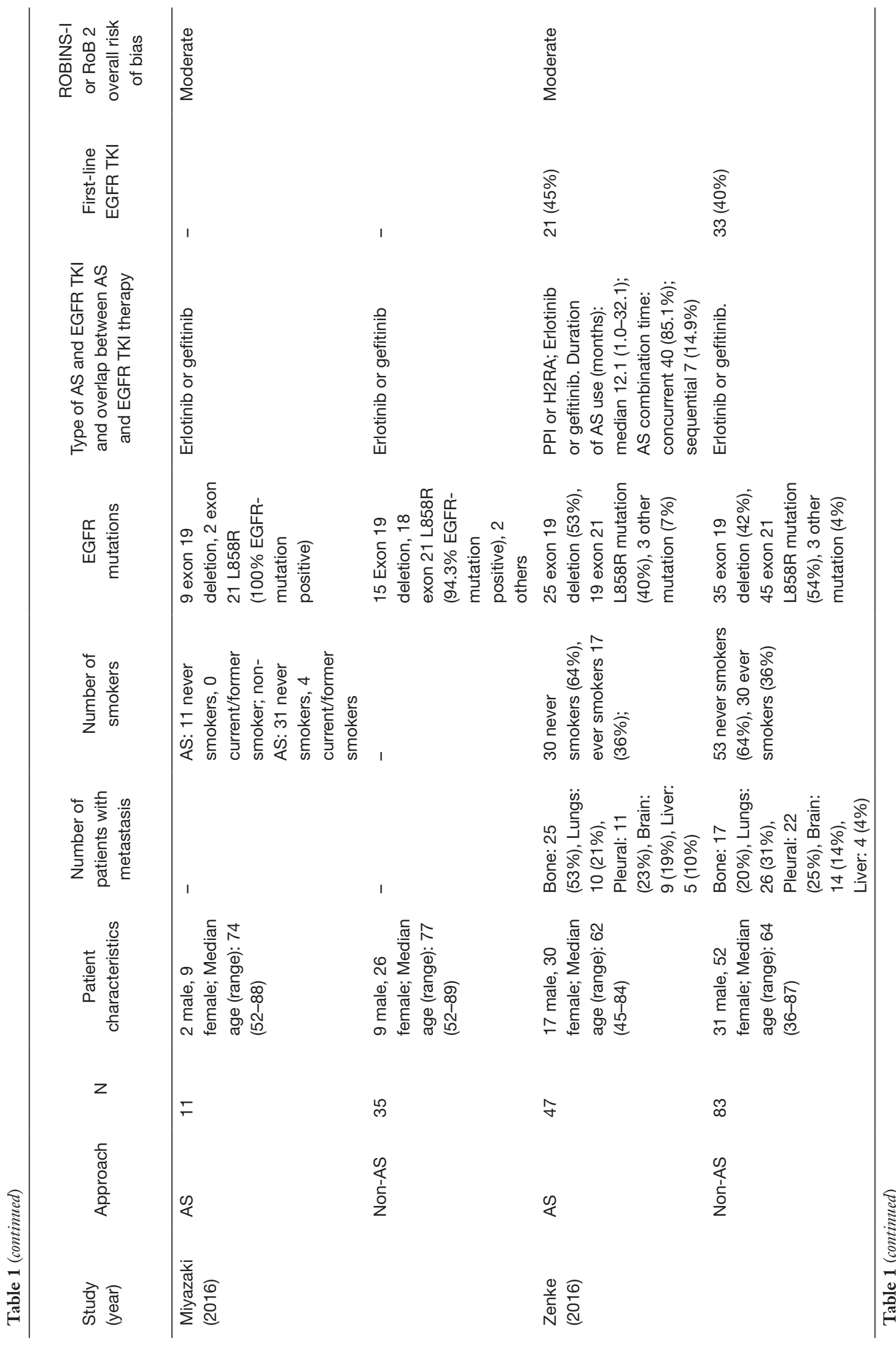




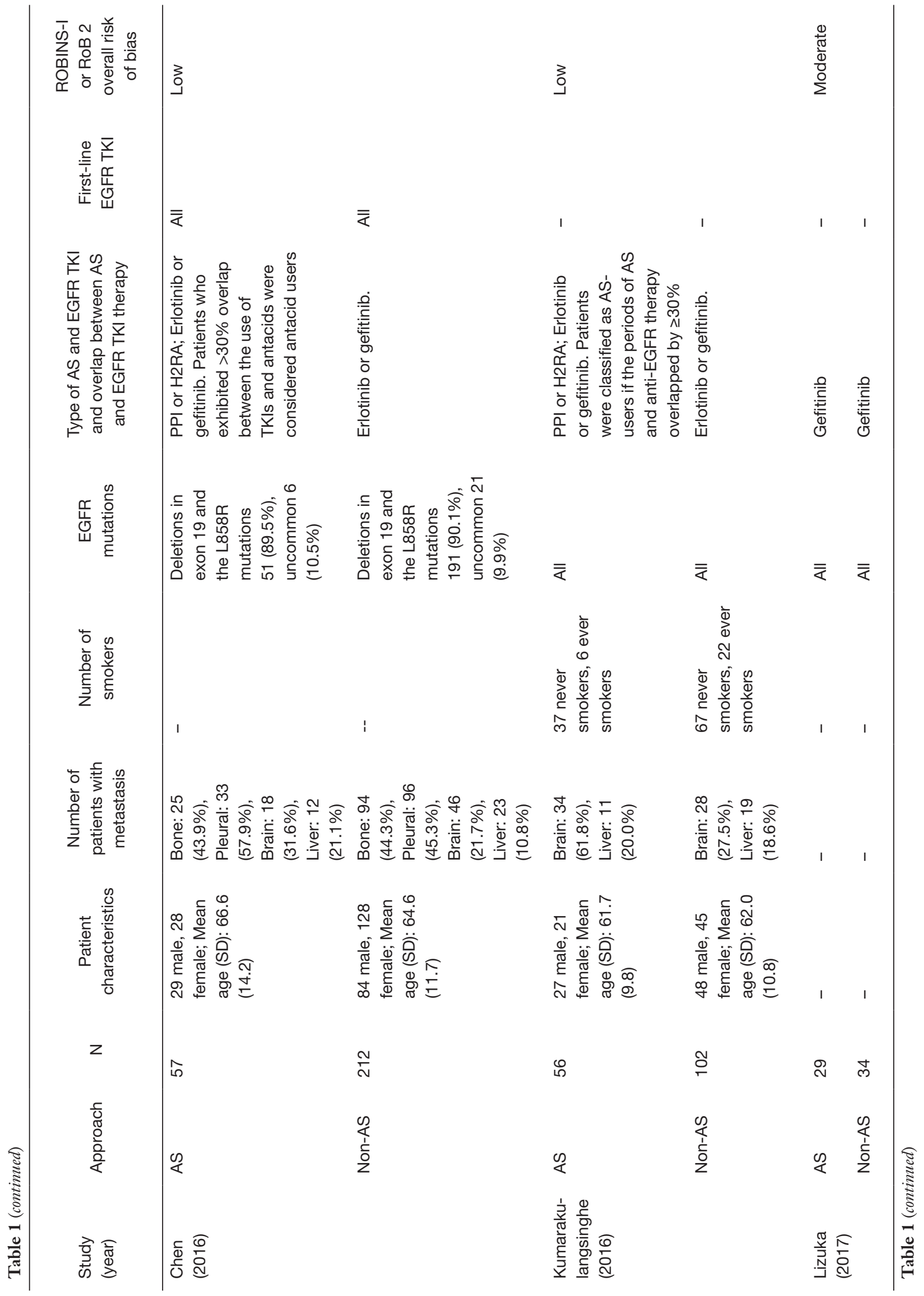




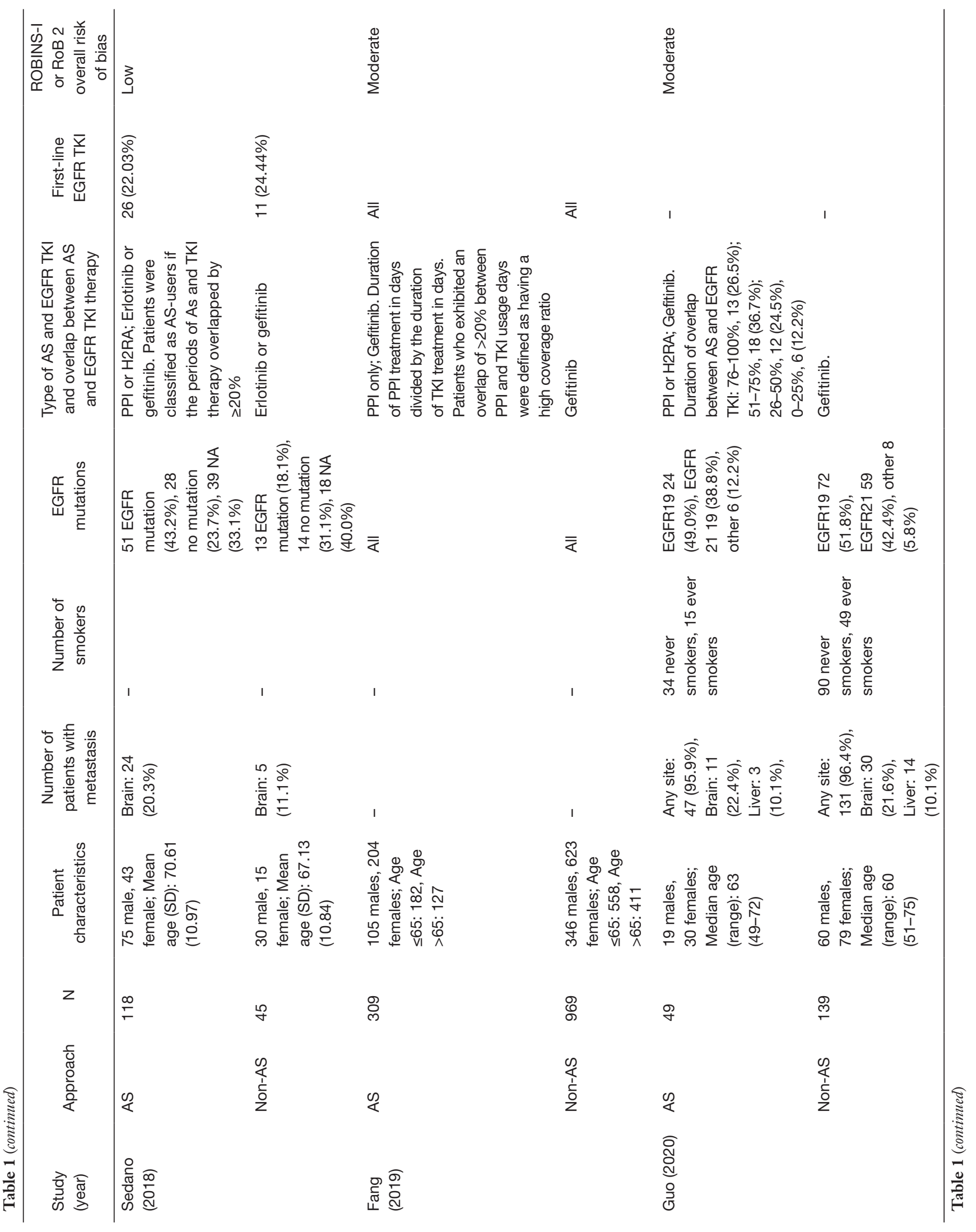




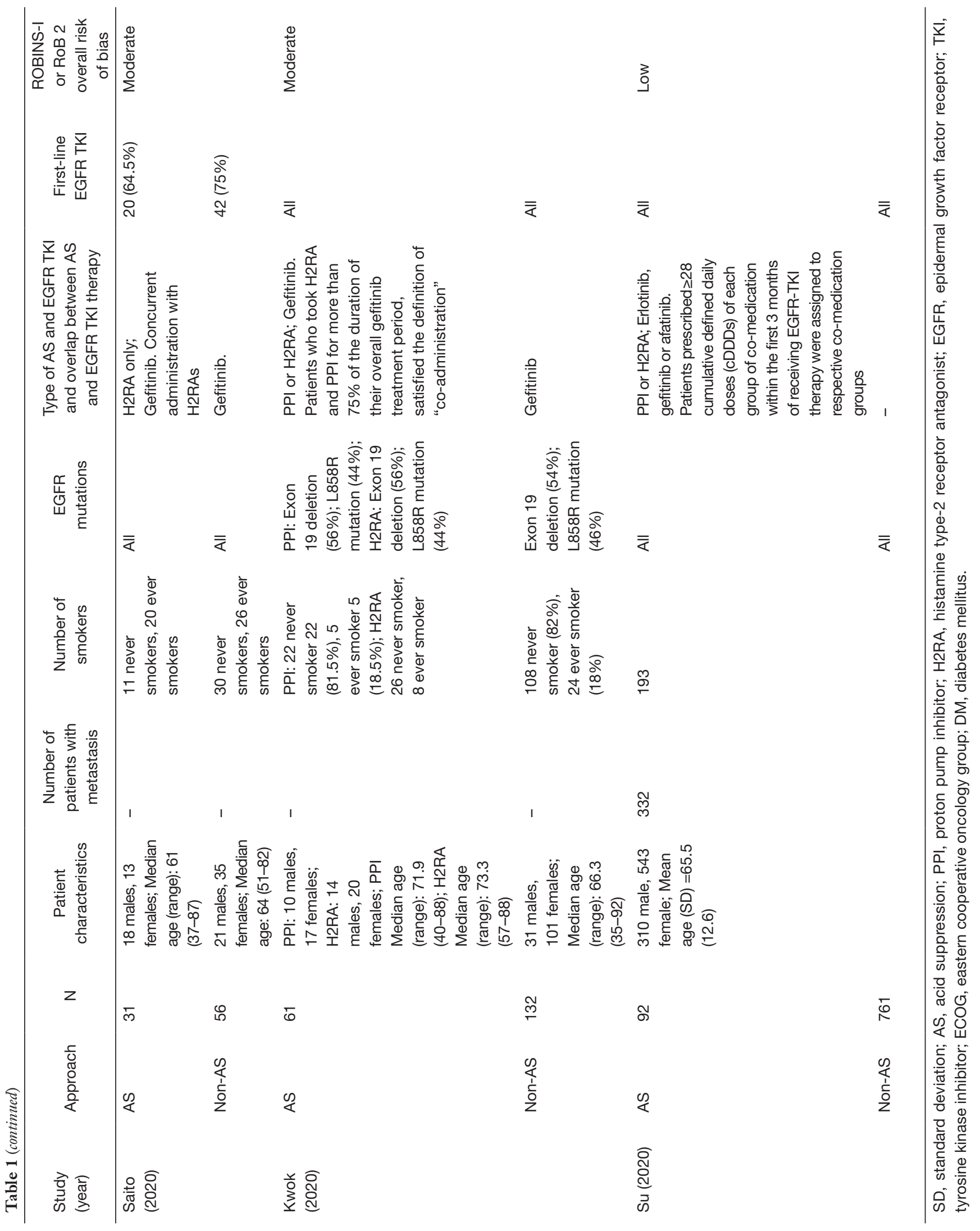




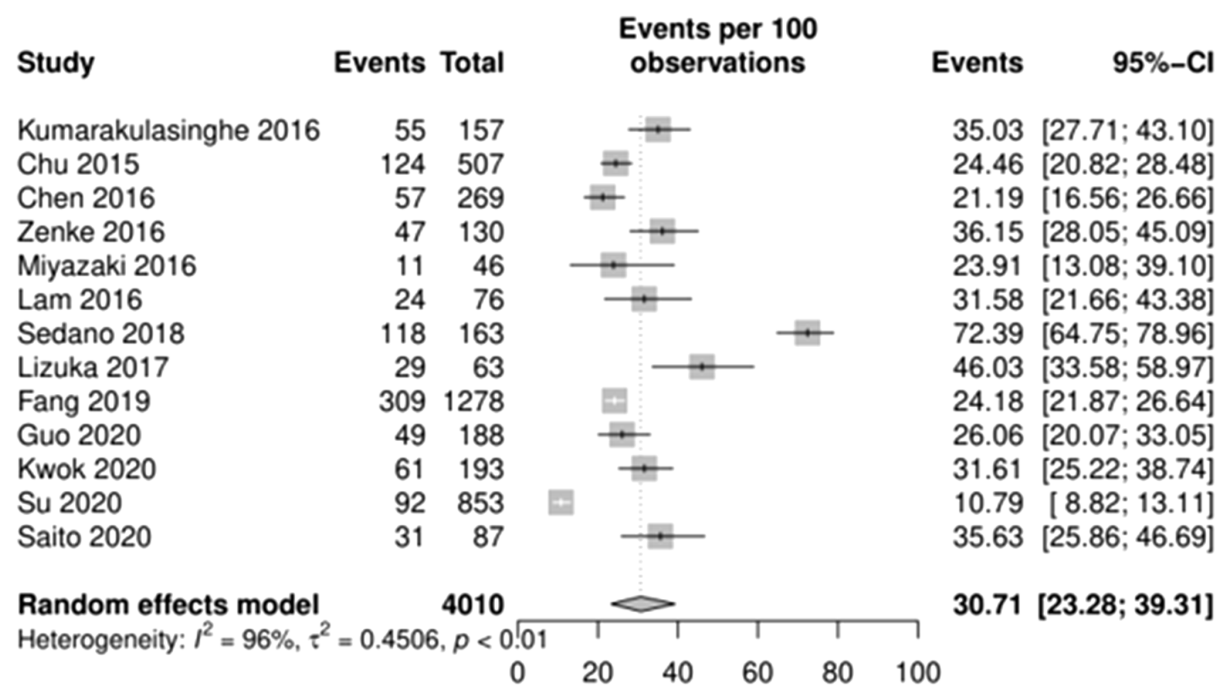

Figure 2 Overall pooled prevalence of AS in patients taking EGFR TKI. AS, acid suppression; EGFR, epidermal growth factor receptor; TKI, tyrosine kinase inhibitor.

not significantly different. However, Hilton et al. reported that the rate of infection was higher in patients with AS (33.7\% AS vs. $20 \%$ non-AS, $\mathrm{P}=0.0008$ ) (29). The summary of adverse effects can be found in Table S2.

\section{Discussion}

With the prevalence of AS use in cancer patients to treat gastric irritation (14) and the effect of AS in EGFR-TKI therapy (8), it is vital to study the interactions between AS drugs and EGFR-TKIs in NSCLC patients. This metaanalysis of high quality retrospective studies comparing the use of EGFR-TKIs with and without AS has shown that AS was strongly associated with poor OS and PFS outcomes. In our study, $30.71 \%$ of predominately NSCLC patients were concurrently treated with AS drugs. OS (HR $=1.46,95 \%$ CI: $1.27-1.67 ; \mathrm{P}<0.00001 ;$ Figure 3$)$ and $\mathrm{PFS}(\mathrm{HR}=1.63$, 95\% CI: 1.35-1.98; P<0.00001; Figure 4) both favored patients treated with EGFR TKI only.

Similar to a previous meta-analysis on the impact of concurrent administration of EGFR TKIs and AS on various cancers (33), this meta-analysis found better survival outcomes for OS and PFS when only EGFRTKIs were used. When combined with AS medications that lower the gastric $\mathrm{pH}$, the alterations in pharmacokinetics of oral EGFR-TKIs led to a decrease in absorption and bioavailability of these drugs, possibly increasing the risk of disease progression and eventually poorer survival.
This is despite no significant differences in the adverse reactions including rash $(\mathrm{OR}=0.81,95 \% \mathrm{CI}: 0.50-1.32$; $\mathrm{P}=0.40)$ and diarrhoea $(\mathrm{OR}=1.03,95 \% \mathrm{CI}: 0.63-1.67$; $\mathrm{P}=0.91)$. However, it is to be noted that those on AS might concurrently be on other medications due to other comorbidities that have an unknown impact on the EGFR TKIs in the NSCLC patient. These comorbidities may also have an adverse effect on the survival of the patient, leading to a decreased OS and PFS in those on AS. With the frequency of over-prescribing AS for gastric issues, it might prove to be valuable to practice caution when prescribing these medications for patients on EGFR-TKI for NSCLC.

However, the effect sizes in this study may be underestimated as some of the included studies analyzed patients with wild-type EGFR or EGFR of unknown mutational status (34-36). EGFR mutation positive patients have been reported to be 10 to 50 -fold more sensitive to gefitinib $(37,38)$. Moreover, some studies included patients receiving afatinib $(32,39)$, which has an absorption that has not been shown to be affected by AS. The sensitivity analysis performed for EGFR mutation positive patients suggests a larger effect size for PFS but there was a lack of appreciable difference in OS potentially due to the use of erlotinib and afatinib in Su et al. (39). Additionally, most of the included studies analyzed patients receiving gefitinib which could be less affected than erlotinib since erlotinib is recommended to be taken with food and AS medications are usually taken before food as well (8). However, this 


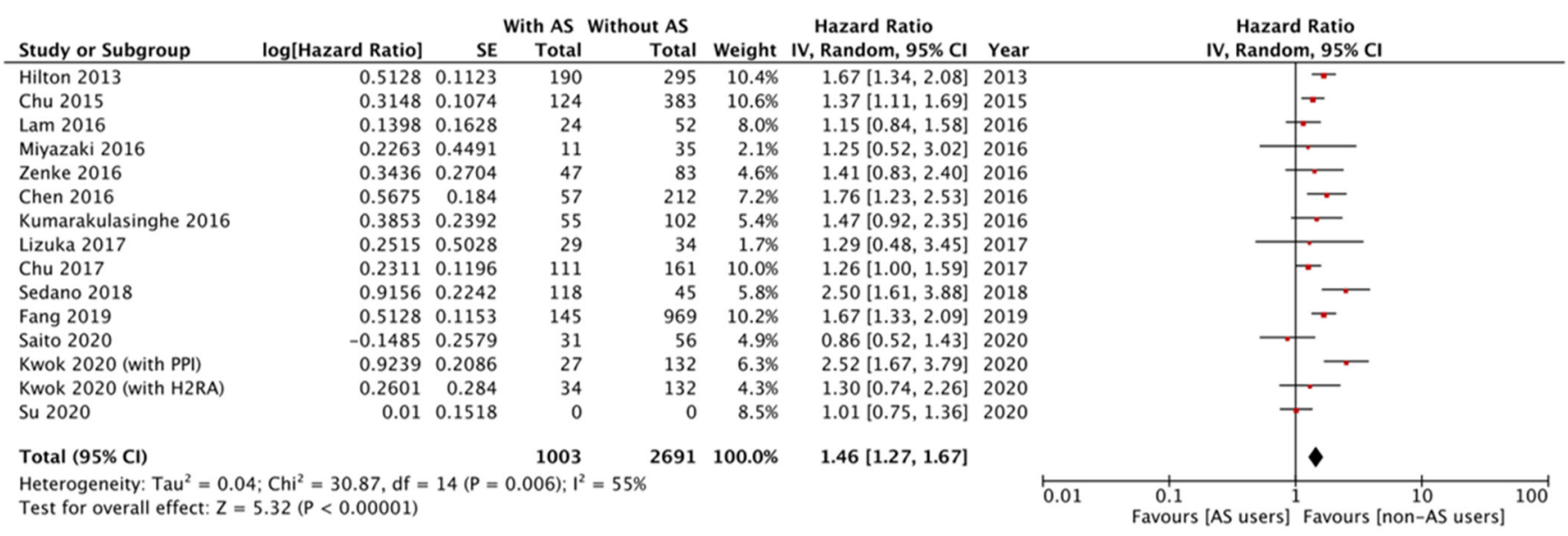

Figure 3 Forest plot for OS. OS, overall survival; AS, acid suppression.

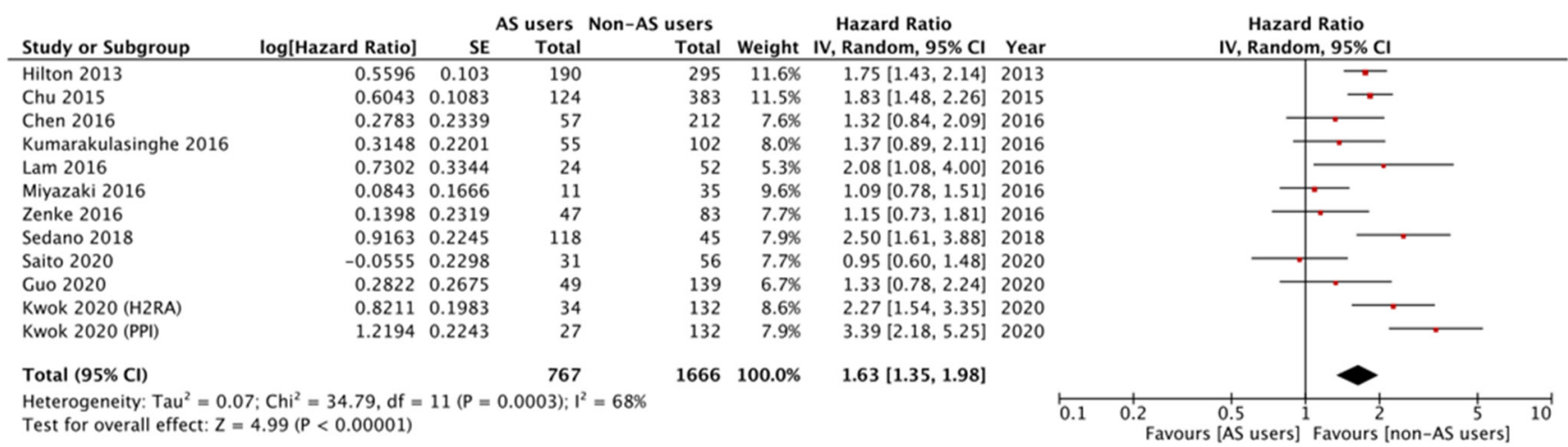

Figure 4 Forest plot for PFS. PFS, progression-free survival; AS, acid suppression.

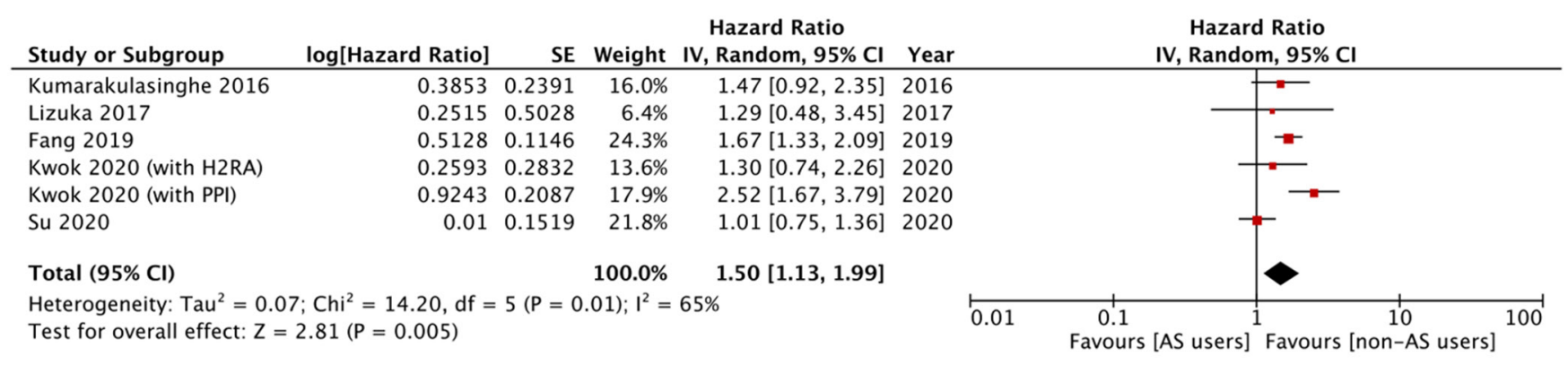

Figure 5 Forest plot for OS sensitivity analysis of EGFR-mutation positive patients. OS, overall survival; EGFR, epidermal growth factor receptor; AS, acid suppression.

point is not to be overstated since AS medications such as PPI achieve long duration of suppression.

Interestingly, clinical outcomes were observed to be dependent on the inverse relationship between levels of
AS and the plasma levels of EGFR TKIs. van Leeuwen et al. found that AUC of erlotinib decreased by $15 \%$ to $33 \%$ when ranitidine dosage was increased from 150 to $300 \mathrm{mg}$ and the AUC decreased even further by 46\% when 


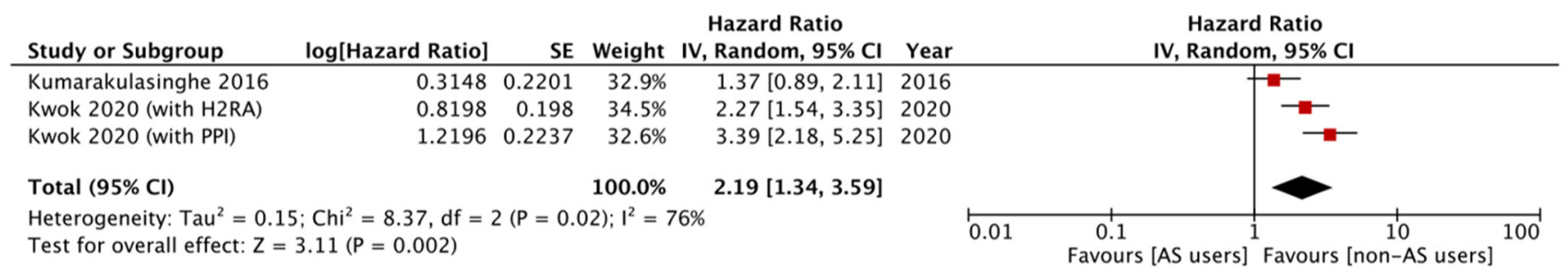

Figure 6 Forest plot for PFS sensitivity analysis of EGFR-mutation positive patients. PFS, progression-free survival; EGFR, epidermal growth factor receptor; AS, acid suppression.

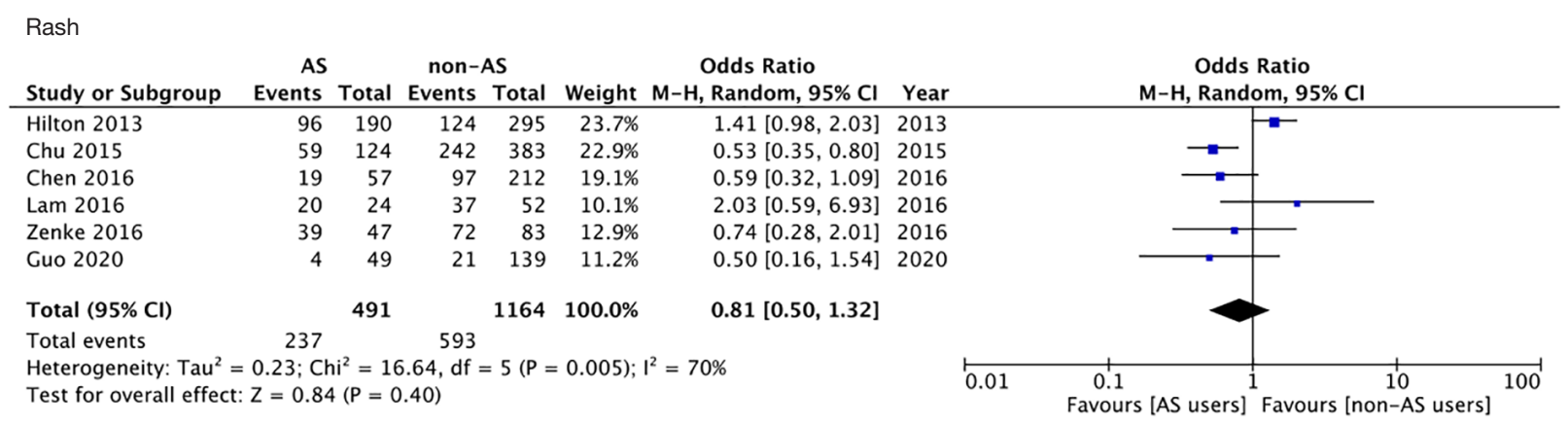

Diarrhoea

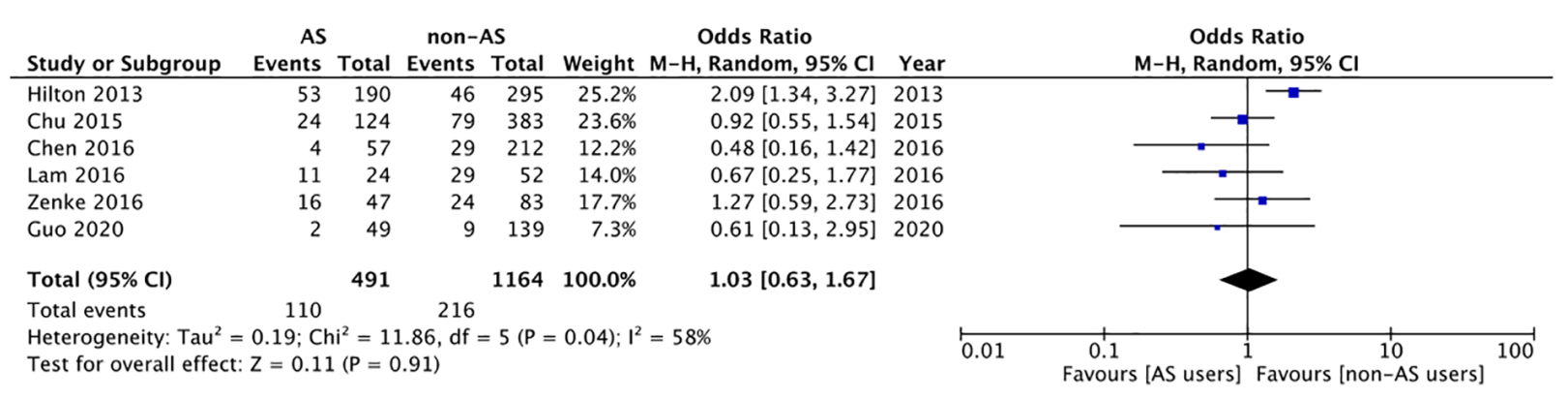

Figure 7 Forest plot for adverse effects. AS, acid suppression.

omeprazole was given (40). In our subgroup analysis of PPI and H2RA, PPI resulted in significantly worse OS and PFS and there was also a significant difference between PPI's and H2RA's effect on OS. H2RA did not significantly worsen both OS and PFS and seems to have a limited impact on the efficacy of EGFR TKI. In the study by Fang et al. (41), higher coverage of PPI resulted in poorer OS and PFS (high coverage HR: 1.67, $\mathrm{P}<0.001$; low coverage ratio HR: 1.29 , $\mathrm{P}=0.027$ ). Similarly, Kwok et al. (42) also found that PPI had stronger acid suppressive effects had a negative impact on OS and PFS and H2RA only had appreciable negative effect on PFS. Potentially, the strength of AS and duration of action has a linear relationship with efficacy of EGFR TKI.
Correspondingly, H2RAs which has a shorter duration of action and achieves a lower intragastric $\mathrm{pH}$ than PPIs has not been shown to negatively affect EGFR TKI efficacy in the study by Saito et al. (43). While it can be hypothesised that only PPI has an impact on the efficacy of EGFR TKI, the subgroup analysis is limited by its small sample size and warrants further investigation into how the strength of AS affects EGFR TKI efficacy.

As EGFR TKIs continue to be used as first line in the treatment of NSCLC and AS drugs continue to be prescribed for various conditions, it is vital to explore the impact on long-term outcomes and safety profile of these drug-drug interactions. There is also limited data available 
on the pharmacokinetics of the dose-dependent reactions of AS on patients taking EGFR-TKIs, hence such data is necessary to find out whether a lower dose of AS may reduce gastrointestinal discomfort while also having an insignificant impact on therapeutic outcomes.

The findings of this study are limited to NSCLC patients receiving first-generation EGFR TKIs (erlotinib and gefitinib). The clinical effect of AS on afatinib efficacy has not been delineated and past studies have also reported that afatinib remained soluble across a wide $\mathrm{pH}$ range of 1-7 (33). Other EGFR TKIs such as second-generation dacomitinib and third generation osimertinib were also not analyzed in this study. Osimertinib is part of the current first-line therapy for advanced NSCLC patients with EGFR-sensitizing mutations and the pharmacokinetics of osimertinib has not been shown to be affected by AS. When compared to taking osimertinib alone, the AUC and Cmax of taking with omeprazole gave a geometric least-squares mean ratio of $106.05 \%$ and $92.75 \%$ respectively with the confidence intervals falling within the equivalence limits of $80-125 \%$ (6). Despite the potential effectiveness of osimertinib in patients concurrently treated with AS medications, the use of 1 st generation EGFR TKIs (erlotinib and gefitinib) as initial treatment is still ubiquitous in many parts of the world due to its comparative affordability and accessibility. There was also an inability to perform subgroup analysis based on the type of EGFR TKI due to insufficient granularity on the oncological agent adopted in the included studies. Furthermore, as an intrinsic limitation of retrospective studies, AS therapy was not randomized and heterogeneity could be introduced in terms of patient baseline characteristics such as performance status, smoking history, histology of NSCLC and presence of metastasis which were not fully controlled for since slightly different covariates were being adjusted for across the studies.

In summary, this meta-analysis delineates that coadministration of $1^{\text {st }}$ generation EGFR-TKIs in NSCLC and AS medications worsens therapeutic outcomes, with a significantly better OS and PFS with only EGFRTKIs. However, more data is needed regarding specific AS drugs, the characteristics of the cancer, and possible dose dependent reactions that AS medications may have on survival outcomes and the side effects. In current practice, physicians should err on the side of caution when prescribing patients undergoing anti-cancer treatment with EGFR-TKIs for NSCLC, and only prescribe such medications when absolutely clinically indicated and opt for a lower strength of AS.

\section{Acknowledgments}

Funding: This research was supported by the National Research Foundation Singapore and the Singapore Ministry of Education under its Research Centres of Excellence initiative. Lingzhi Wang is supported by Joint NCIS and NUS Cancer Program Seed Funding Grants/2020, National University of Singapore. Boon Cher Goh is supported by the National Medical Research Council, Singapore (NMRC/CSA-SI/0006/2016 and NMRC/CG/ M005/2017_NCIS).

\section{Footnote}

Reporting Checklist: The authors have completed the PRISMA reporting checklist. Available at https://dx.doi. org/10.21037/tlcr-21-378

Conflicts of Interest: All authors have completed the ICMJE uniform disclosure form (available at https://dx.doi. org/10.21037/tlcr-21-378). LW is supported by Joint National University Cancer Institute, Singapore (NCIS) and NUS Cancer Program Seed Funding Grants/2020, National University of Singapore. BCG is supported by the National Medical Research Council, Singapore (NMRC/ CSA-SI/0006/2016 and NMRC/CG/M005/2017_NCIS). RS is part of the advisory board for Astra-Zeneca, AMGEN, BMS, Boehringer Ingelheim, Lilly, Merck, Novartis, Pfizer, Roche, Taiho, Takeda, and Yuhan; and research funding from Astra-Zeneca and Boehringer Ingelheim, outside the submitted work. The other authors have no conflicts of interest to declare.

Ethical Statement: The authors are accountable for all aspects of the work in ensuring that questions related to the accuracy or integrity of any part of the work are appropriately investigated and resolved.

Open Access Statement: This is an Open Access article distributed in accordance with the Creative Commons Attribution-NonCommercial-NoDerivs 4.0 International License (CC BY-NC-ND 4.0), which permits the noncommercial replication and distribution of the article with the strict proviso that no changes or edits are made and the original work is properly cited (including links to both the formal publication through the relevant DOI and the license). 
See: https://creativecommons.org/licenses/by-nc-nd/4.0/.

\section{References}

1. Sung H, Ferlay J, Siegel RL, et al. Global Cancer Statistics 2020: GLOBOCAN Estimates of Incidence and Mortality Worldwide for 36 Cancers in 185 Countries. CA Cancer J Clin 2021;71:209-49.

2. Rusch V, Klimstra D, Venkatraman E, et al. Overexpression of the epidermal growth factor receptor and its ligand transforming growth factor alpha is frequent in resectable non-small cell lung cancer but does not predict tumor progression. Clin Cancer Res 1997;3:515-22.

3. National Comprehensive Cancer Network. Non-Small Cell Lung Cancer (Version 4.2021). Available online: https://www.nccn.org/professionals/physician_gls/pdf/ nscl.pdf. Accessed: April 22, 2021. 2021.

4. Zhou C, Wu YL, Chen G, et al. Erlotinib versus chemotherapy as first-line treatment for patients with advanced EGFR mutation-positive non-small-cell lung cancer (OPTIMAL, CTONG-0802): a multicentre, open-label, randomised, phase 3 study. Lancet Oncol 2011;12:735-42.

5. Rosell R, Carcereny E, Gervais R, et al. Erlotinib versus standard chemotherapy as first-line treatment for European patients with advanced EGFR mutation-positive non-small-cell lung cancer (EURTAC): a multicentre, open-label, randomised phase 3 trial. Lancet Oncol 2012;13:239-46.

6. Vishwanathan K, Dickinson PA, Bui K, et al. The Effect of Food or Omeprazole on the Pharmacokinetics of Osimertinib in Patients With Non-Small-Cell Lung Cancer and in Healthy Volunteers. J Clin Pharmacol 2018;58:474-84.

7. Ohgami M, Kaburagi T, Kurosawa A, et al. Effects of Proton Pump Inhibitor Coadministration on the Plasma Concentration of Erlotinib in Patients With Non-Small Cell Lung Cancer. Ther Drug Monit 2018;40:699-704.

8. Budha NR, Frymoyer A, Smelick GS, et al. Drug absorption interactions between oral targeted anticancer agents and PPIs: is $\mathrm{pH}$-dependent solubility the Achilles heel of targeted therapy? Clin Pharmacol Ther 2012;92:203-13.

9. Scarpignato C, Gatta L, Zullo A, et al. Effective and safe proton pump inhibitor therapy in acid-related diseases - A position paper addressing benefits and potential harms of acid suppression. BMC Med 2016;14:179.
10. Welage LS. Overview of pharmacologic agents for acid suppression in critically ill patients. Am J Health Syst Pharm 2005;62:S4-S10.

11. Schubert ML, Peura DA. Control of gastric acid secretion in health and disease. Gastroenterology 2008;134:1842-60.

12. Tofil NM, Benner KW, Fuller MP, et al. Histamine 2 receptor antagonists vs intravenous proton pump inhibitors in a pediatric intensive care unit: a comparison of gastric pH. J Crit Care 2008;23:416-21.

13. Parente F, Cucino C, Gallus S, et al. Hospital use of acidsuppressive medications and its fall-out on prescribing in general practice: a 1-month survey. Aliment Pharmacol Ther 2003;17:1503-6.

14. Smelick GS, Heffron TP, Chu L, et al. Prevalence of acid-reducing agents (ARA) in cancer populations and ARA drug-drug interaction potential for molecular targeted agents in clinical development. Mol Pharm 2013;10:4055-62.

15. Liberati A, Altman DG, Tetzlaff J, et al. The PRISMA statement for reporting systematic reviews and metaanalyses of studies that evaluate healthcare interventions: explanation and elaboration. BMJ 2009;339:b2700.

16. Wan X, Wang W, Liu J, et al. Estimating the sample mean and standard deviation from the sample size, median, range and/or interquartile range. BMC Med Res Methodol 2014;14:135.

17. Hozo SP, Djulbegovic B, Hozo I. Estimating the mean and variance from the median, range, and the size of a sample. BMC Med Res Methodol 2005;5:13.

18. Parmar MK, Torri V, Stewart L. Extracting summary statistics to perform meta-analyses of the published literature for survival endpoints. Stat Med 1998;17:2815-34.

19. Schwarzer G, Chemaitelly H, Abu-Raddad LJ, et al. Seriously misleading results using inverse of FreemanTukey double arcsine transformation in meta-analysis of single proportions. Res Synth Methods 2019;10:476-83.

20. Clopper CJ, Pearson ES. The use of confidence or fiducial limits illustrated in the case of the binomial. Biometrika 1934;26:404-13.

21. Fletcher J. What is heterogeneity and is it important? BMJ 2007;334:94-6.

22. DerSimonian R, Laird N. Meta-analysis in clinical trials. Control Clin Trials 1986;7:177-88.

23. Sterne JA, Sutton AJ, Ioannidis JP, et al. Recommendations for examining and interpreting funnel plot asymmetry in meta-analyses of randomised controlled trials. BMJ 2011;343:d4002. 
24. Sedgwick $\mathrm{P}$. What is publication bias in a meta-analysis? BMJ 2015;351:h4419.

25. Sterne JAC, Savović J, Page MJ, et al. RoB 2: a revised tool for assessing risk of bias in randomised trials. BMJ 2019;366:14898.

26. Sterne JA, Hernán MA, Reeves BC, et al. ROBINS-I: a tool for assessing risk of bias in non-randomised studies of interventions. BMJ 2016;355:i4919.

27. Chu MP, Hecht JR, Slamon D, et al. Association of Proton Pump Inhibitors and Capecitabine Efficacy in Advanced Gastroesophageal Cancer: Secondary Analysis of the TRIO-013/LOGiC Randomized Clinical Trial. JAMA Oncol 2017;3:767-73.

28. Xu ZY, Li JL. Comparative review of drug-drug interactions with epidermal growth factor receptor tyrosine kinase inhibitors for the treatment of non-smallcell lung cancer. Onco Targets Ther 2019;12:5467-84.

29. Hilton JF, Tu D, Seymour L, et al. An evaluation of the possible interaction of gastric acid suppressing medication and the EGFR tyrosine kinase inhibitor erlotinib. Lung Cancer 2013;82:136-42.

30. Zenke Y, Yoh K, Matsumoto S, et al. Clinical Impact of Gastric Acid-Suppressing Medication Use on the Efficacy of Erlotinib and Gefitinib in Patients With Advanced Non-Small-Cell Lung Cancer Harboring EGFR Mutations. Clin Lung Cancer 2016;17:412-8.

31. Guo Z. Concomitant administration of gastric acid suppression might attenuates the clinical efficacy of gefitinib: a single cancer center retrospective study. J Chin Pharm Sci 2020;29:192-8.

32. Chen YM, Lai CH, Chang HC, et al. Antacid Use and De Novo Brain Metastases in Patients with Epidermal Growth Factor Receptor-Mutant Non-Small Cell Lung Cancer Who Were Treated Using First-Line First-Generation Epidermal Growth Factor Receptor Tyrosine Kinase Inhibitors. PLoS One 2016;11:e0149722.

33. Indini A, Petrelli F, Tomasello G, et al. Impact of Use of Gastric-Acid Suppressants and Oral Anti-Cancer Agents on Survival Outcomes: A Systematic Review and MetaAnalysis. Cancers (Basel) 2020;12:998.

34. Chu MP, Ghosh S, Chambers CR, et al. Gastric Acid suppression is associated with decreased erlotinib efficacy in non-small-cell lung cancer. Clin Lung Cancer 2015;16:33-9.

35. Lam LH, Capparelli EV, Kurzrock R. Association of concurrent acid-suppression therapy with survival outcomes and adverse event incidence in oncology patients receiving erlotinib. Cancer Chemother Pharmacol 2016;78:427-32.

36. Nieves Sedano M, Manuel Caro Teller J, García Muñoz C, et al. Clinical impact of gastric acid suppressing medication on the effectiveness of tyrosine kinase inhibitors in lung cancer patients. J BUON 2018;23:647-53.

37. Paez JG, Jänne PA, Lee JC, et al. EGFR mutations in lung cancer: correlation with clinical response to gefitinib therapy. Science 2004;304:1497-500.

38. Lynch TJ, Bell DW, Sordella R, et al. Activating mutations in the epidermal growth factor receptor underlying responsiveness of non-small-cell lung cancer to gefitinib. N Engl J Med 2004;350:2129-39.

39. Su VY, Yang KY, Huang TY, et al. The efficacy of first-line tyrosine kinase inhibitors combined with co-medications in Asian patients with EGFR mutation non-small cell lung cancer. Sci Rep 2020;10:14965.

40. van Leeuwen RW, van Gelder T, Mathijssen RH, et al. Drug-drug interactions with tyrosine-kinase inhibitors: a clinical perspective. Lancet Oncol 2014;15:e315-26.

41. Fang YH, Yang YH, Hsieh MJ, et al. Concurrent proton-pump inhibitors increase risk of death for lung cancer patients receiving 1st-line gefitinib treatment - a nationwide population-based study. Cancer Manag Res 2019;11:8539-46.

42. Kwok WC, Ho JC, Lam DC, et al. Clinical Impact of Gastric Acid Suppressants Use on the Efficacy of Gefitinib in Patients with Advanced Adenocarcinoma of the Lung Harboring Common EGFR Mutations. Clin Cancer Drugs 2020;7:57-61.

43. Saito Y, Takekuma Y, Kobayashi M, et al. Impact of histamine type-2 receptor antagonists on the anticancer efficacy of gefitinib in patients with non-small cell lung cancer. Eur J Clin Pharmacol 2021;77:381-8.

Cite this article as: Sim W, Jain SR, Lim WH, Chin YH, Ng CH, Syn N, Goh KS, Soo R, Wang L, Goh BC. Interactions between epidermal growth factor receptor tyrosine kinase inhibitors and proton-pump inhibitors/histamine type2 receptor antagonists in non-small cell lung cancer: a systematic review and meta-analysis. Transl Lung Cancer Res 2021;10(8):3567-3581. doi: 10.21037/tlcr-21-378 


\section{Supplementary}

Table S1 Search strategy for Medline

1: Exp ErbB Receptors/ai [antagonists and inhibitors] or Exp Protein Kinase Inhibitors/

2: (((epidermal growth factor receptor* or egfr or tyrosine kinase or erb* or her1) adj inhibitor*) or erlotinib or gefitinib or afatinib or dacomitinib or osimertinib or weak base drug*).tw.

3: 1 or 2

4: Exp Proton Pump Inhibitors/ or Exp Histamine H2 Antagonists/ or Exp Omeprazole/ or Exp Gastric Acid/

5: (acid reduc* or acid suppress* or $\mathrm{PPI}^{\star}$ or histamine h2 receptor antagonist* or h2 blocker* or omeprazole or antacid ${ }^{\star}$ ).tw.

6: 4 or 5

7: Exp Drug Interactions/

8: (effect* ${ }^{\star}$ or efficac ${ }^{\star}$ or toxic* or impact $^{\star}$ or interaction* or $\left.^{\text {ddi }}{ }^{\star}\right)$.tw.

9: 7 or 8

10: 3 and 6 and 9

Table S2 Summary of adverse effects

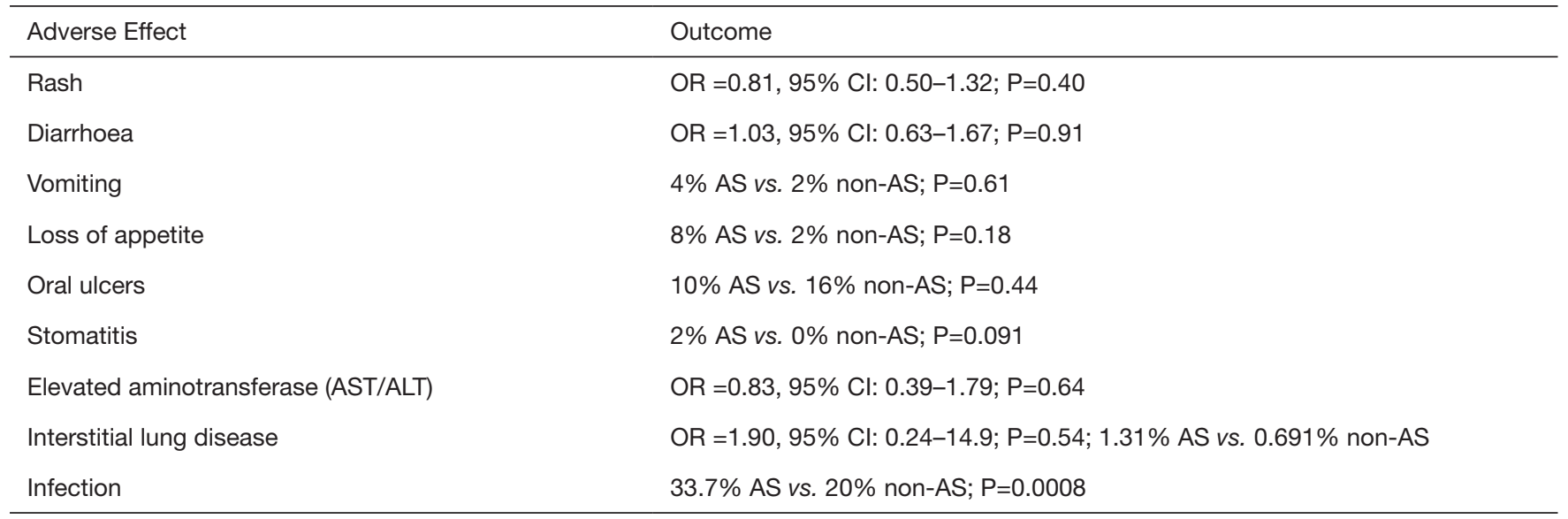

OR, odds ratio; AST, aspartate aminotransferase; ALT, alanine aminotransferase. 


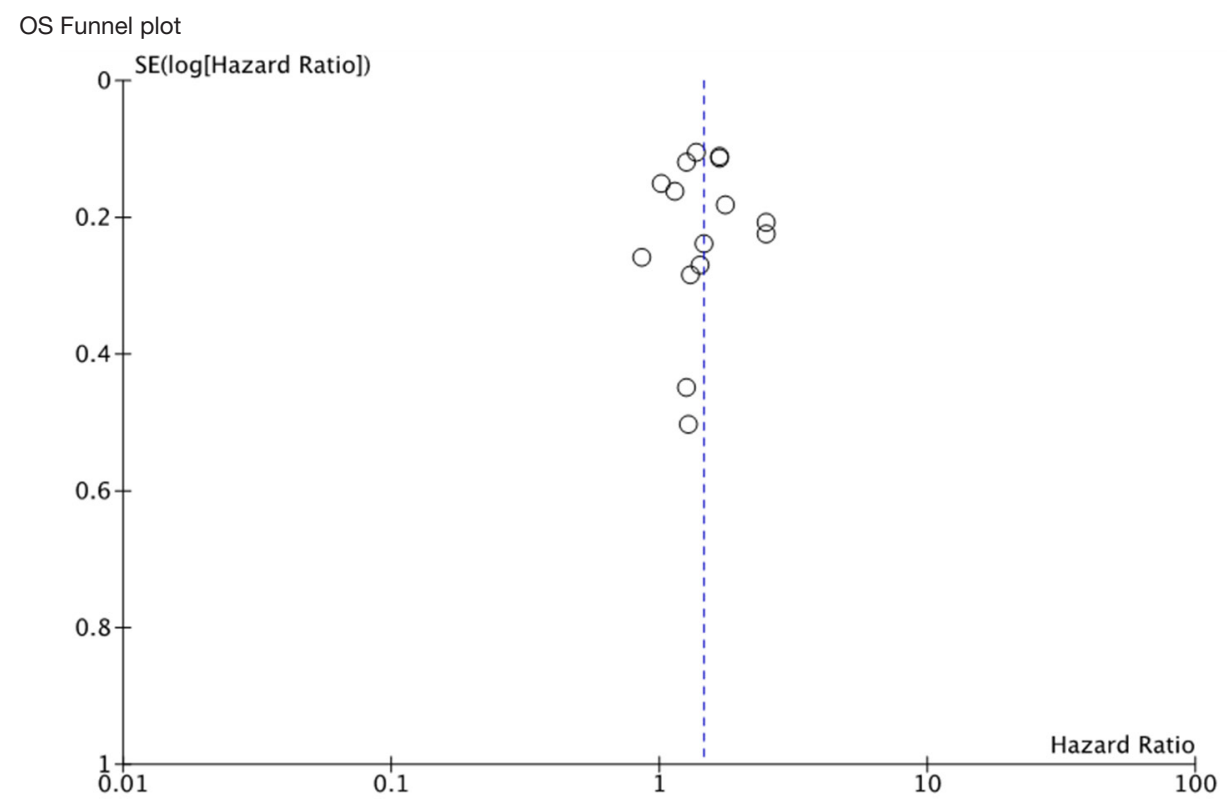

PFS Funnel plot

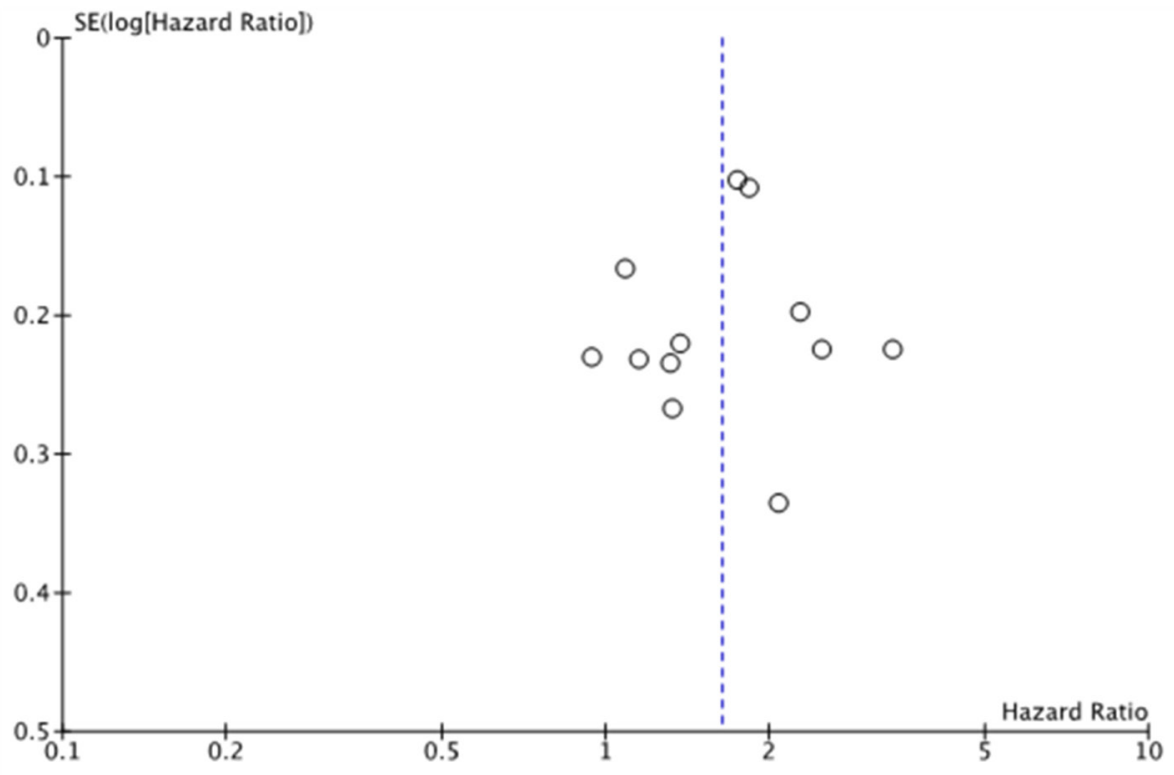

Figure S1 Funnel plots. 